\title{
Formally mapping the typologies of interacting ABCD systems
}

\author{
Wm. G. Bennett, ${ }^{1}$ Natalie DelBusso, ${ }^{2}$ and Luca Iacoponi ${ }^{2}$ \\ ${ }^{I}$ Rhodes University and ${ }^{2}$ Rutgers University
}

\section{ABCs of ABCD}

The theory of Agreement By Correspondence has gained prominence as a way to explain harmony patterns, especially long-distance consonant agreement (Rose \& Walker 2004, Hansson 2010). Bennett (2015) observes that the theory also generates Dissimilation, even with no further assumptions made (ergo 'ABCD'). This connection between dissimilation and assimilation is an appealing result, as it was in a large body of previous work that draws on the same mechanisms for both kinds of patterns (Mester 1986, Yip 1988, etc.).

Recent work along ABCD lines has developed a range of varying formalizations. One point of variation is the correspondence relation at the heart of the theory, and the formal properties of it: is all correspondence homogenous? Is the relation transitive? Is it symmetric? Accompanying such questions are differences in the formal character of the constraints that refer to the correspondence relation: are agreement violations calculated over whole forms, or locally, based on pairs of correspondence? These points of difference definitively affect the typologies that result - particularly for situations where correspondence and/or agreement constraints based on multiple different features may conflict. Previous work has demonstrated as much through analyses of specific cases that seem to work far better in one version than others. ${ }^{1}$ But the analysis of individual case studies is not the most pressing question for the modern theorist: the much more important question is which of these different $\mathrm{ABCD}$ formulations makes the right typological predictions. Any answer to that question presupposes that we know what the typological predictions are. This is far from simple: all of the various formulations of ABCD are intended to generalize across different features, resulting in fairly large sets of constraints. Moreover, demonstrating all the effects of such constraints requires consideration of multiple segmental forms, with multiple correspondence structures available for each. The result is that we are comparing systems that are sufficiently large that their predictions cannot be deduced from intuition alone.

This paper takes a step towards that goal. Our aim is to understand the interaction of two ABCD subsystems. Breaking the theory down into sub-systems, helpfully, models a key point of interest in comparing competing formulations. Many of the known differences between different $\mathrm{ABCD}$ formulations emerge from the interaction of two distinct $\mathrm{ABCD}$ effects (='ABCDE's: harmony or dissimilation patterns). So, if we want to understand the full predictions of any $A B C D$ theory, we must know what possibilities it admits for the relationship between two harmony/dissimilation systems based on different features.

The rest of the paper is organized in the following way. Section 2 defines the sub-systems analyzed here - the candidates and constraints included in each. These are modeled on a real-world point of departure, in the form of Kinyarwanda - a language with harmony among sibilants, and dissimilation between voiceless obstruents that straddle the edge of the stem (see Bennett 2015 and sources therein for details). We analogize this with one sub-system capable of showing the voiceless dissimilation pattern, and

\footnotetext{
${ }^{*}$ For useful feedback and discussion, thanks are due to Alan Prince, Birgit Abler, Nazarré Merchant, Rachel Walker, Nick Danis, Paula Houghton, Eric Baković, Arto Anttila, Jason Riggle, Stephanie Shih, Gunnar Hansson, as well as to audiences and bystanders at the 2015 Annual Meeting in Phonology in Vancouver, and the Workshop on Formal Typologies in OT at Rutgers. This work was supported in part by a grant from Rhodes University. Authors' names are in alphabetical order; any mistakes should be attributed to the first name on the list.

${ }^{1}$ A sampling of the relevant literature includes: Hansson (2007, 2014), Iacoponi (2016), Inkelas \& Shih (2014), Shih \& Inkelas (2014), Walker (2000a, 2000b, 2011, 2015).

(C) 2016 Wm. G. Bennett, Natalie DelBusso, and Luca Iacoponi

Proceedings of AMP 2015

Completed April 7, 2016
} 
another capable of exhibiting sibilant harmony. Sections 3 and 4 present the full typologies of these two fragments of the theory. Section 5 presents their interactions: the typology predicted when the two very simple fragments are combined into a larger system. Section 6 analyzes the typologies into properties (Alber, Delbusso \& Prince 2015, Merchant \& Prince 2015, Alber \& Prince forthcoming), and remarks on the relationship between the properties of the simple fragments and their containment in the larger system. Section 7 reiterates the conclusion: that the predictions of a complex ABCD system are understandable as the interaction of much simpler sub-systems.

\section{Defining ABCD systems and subsystems}

In the interests of starting from the simple and building out to the complex, we consider three $A B C D$ constraint systems: two are very simple, and the third is their union. These systems are defined here.

2.1 GEN_2rt2f We consider a fairly narrow GEN, which is common to all three of systems considered here. All inputs and candidates have the structure in (1). The label ' $2 \mathrm{rt}$ ' alludes to the way such forms represent a single root of vaguely CVCV shape - containing exactly two consonants, with a syllable edge in between, but no internal morphological complexity. (Since all constraints refer to consonants, the vowels are irrelevant - and are omitted to simplify typography). The segmental inventory available is defined as $\{\mathrm{t}$ $\mathrm{s} \mathrm{d} z\}:$ a total of four segments, with two fully-crossed binary features, [ \pm sibilant] and [ \pm voice].

$$
\text { CV . CV (where } \mathrm{C} \in\{\mathrm{t} \mathrm{s} \mathrm{d} \mathrm{z}\}, \mathrm{V} \text { is an irrelevant vowel, and '.' is a syllable edge) }
$$

The inputs considered for each system are all 16 possible combinations of two consonants: $\{\mathrm{t} \mathrm{t}, \mathrm{t} \mathrm{s}, \mathrm{t} \mathrm{d}, \mathrm{t} \mathrm{z}$, $\ldots, \mathrm{z} \mathrm{z}$. For each input, the space of candidates consists of all of these segmental forms, distinguished by surface correspondence possibilities. This combination of inputs and candidates was used to calculate factorial typologies in OTWorkplace (Prince, Tesar \& Merchant 2015).

The formulation of surface correspondence assumed here is the one proposed by Bennett (2015), which takes it as a homogenous, symmetric, transitive, and reflexive relation. Since all forms contain exactly two consonants, there are exactly two possible surface correspondence structures for each segmental form: the consonants either correspond with one another, or they don't. The space of candidates is thus made up of pairs of competitors that have exactly the same segmental form, but differ in correspondence. This is represented in candidates with indices $\{x y\}$. Thus, $\left\langle s_{x} z_{x}\right\rangle$ represents a candidate with the segmental form [sV.zV], with the surface correspondence structure $\{\mathrm{s} \mathrm{z}\}$ - having correspondence between the two Cs. This competes with alternatives such as $\left\langle s_{x} z_{y}\right\rangle$, which has the same segments but not in correspondence with each other; and also $\left\langle z_{\mathrm{x}} z_{\mathrm{x}}\right\rangle$, which has different segments (the result of harmony for voicing); and with candidates that may differ in segmental form and also correspondence structure, such as $\left\langle t_{x} z_{y}\right\rangle,\left\langle d_{x} s_{y}\right\rangle,\left\langle z_{x} t_{y}\right\rangle$, etc.

In the interests of completeness, we include the full set of candidates as possible output mappings for each input - but we note that many of them have identical violation profiles in one or more of the subsystems considered. This is because the constraints comprising each subsystem are not sensitive to all of the intuitive differences between candidates.

2.2 CON_2rt2f We consider three CON systems here, two of which are subsets of the third. These are defined in (2), with constraints adapted from Bennett's (2015) analysis of Kinyarwanda.

\begin{tabular}{|c|c|c|c|}
\hline & 2rt_VlessDiss & 2rt_SibHarm & 2rt2f \\
\hline $\begin{array}{l}\text { SCorr constraints } \\
\text { (markedness) }\end{array}$ & $\begin{array}{l}\text { CORR } \cdot[- \text { voice }], \\
\text { CC } \cdot \operatorname{EdGE}(\sigma)\end{array}$ & $\begin{array}{l}\text { CORR } \cdot[+ \text { sibilant }], \\
\text { CC } \cdot \text { IDENT(voice) }\end{array}$ & $\begin{array}{l}\text { CORR } \cdot[- \text { voice }], \text { CORR } \cdot[+ \text { sibilant }] \\
\text { CC } \cdot \operatorname{EDGE}(\sigma), \text { CC } \cdot \text { IDENT(voice) }\end{array}$ \\
\hline $\begin{array}{l}\text { I-O faithfulness } \\
\text { constraints }\end{array}$ & IDENT(voice) & $\begin{array}{l}\text { IDENT(voice), } \\
\text { IDENT(sibilant) }\end{array}$ & $\begin{array}{l}\text { IDENT(voice), } \\
\text { IDENT(sibilant) }\end{array}$ \\
\hline Size of typology & $3 \operatorname{lgs}$. & 4 lgs. & 16 lgs. \\
\hline
\end{tabular}


Kinyarwanda exhibits voiceless dissimilation across the edge of the stem domain, and sibilant harmony within the stem. The 2rt_VlessDiss system models the dissimilation process, using the syllable edge as the homolog of the stem edge in Kinyarwanda: the domain edge that conditions dissimilation for segments that straddle it. The 2rt_SibHarm system models the sibilant harmony, with the combination of CC.IDENT(voice) and CORR·[+sibilant] capable of driving voicing agreement among sibilants. The system $2 \mathrm{rt} 2 \mathrm{f}$ is the combination of the two simpler systems. Its typology therefore includes all possible ways in which the simpler $\mathrm{ABCD}$ systems can interact with one another - an opportunity to understand the fundamentals types of interactions that can emerge in an $\mathrm{ABCD}$ theory.

\section{2rt_VlessDiss: typology of a 3-constraint ABCD subsystem}

The 2rt_VlessDiss subsystem consists of only three constraints: CORR $[-$-voice], CC.EDGE $(\sigma)$, and IDENT(voice). Its typology, shown in (3) below, is accordingly quite simple. The rows show the distinct languages arising from all possible rankings of the three constraints, named schematically based on their behavior. The columns show inputs, and the colored cells indicate what each input maps to in each language. The choice of color indicates the type of mapping: gray cells represent faithful candidates with surface correspondence, beige cells are faithful candidates with surface non-correspondence, and blue cells show dissimilation of [-voice].

Only a small subset of the space of possible inputs shows any distinctions at all. In (3), the first two inputs are sufficient to show the full range of possibilities for how inputs are treated in the typology; the third input serves only to illustrate that others are redundant. This happens because the constraints in this sub-system only refer to one feature, namely $[ \pm$ voice $]$; and the only feature-sensitive markedness constraint, CORR $\cdot[-$ voice], refers only to [-voice], specifically. As such, all inputs with one or more voiced consonants invariably surface faithfully with respect to voicing. Since IDENT(sibilant) is not part of the $\mathrm{CON}$ of this sub-system, candidates that differ in [ ${ }$ sibilant] are formally identical - resulting in large groups of co-optima for all inputs. What these bunches of co-optima have in common is their correspondence structure, and distribution of $[ \pm$ voice $]$.

(3) Typology generated by $2 \mathrm{rt}$-VlessDiss sub-system

\begin{tabular}{l|ccc|l}
$\downarrow$ Lgs $\backslash$ Inputs $\rightarrow$ & $\mathbf{t}$ & $\mathbf{t}$ & $\mathbf{s}$ t & Remarks \\
\hline \multirow{4}{*}{ F.cor } & $\mathrm{t}_{\mathrm{x}} \mathrm{t}_{\mathrm{x}}$ & $\mathrm{t}_{\mathrm{x}} \mathrm{d}_{\mathrm{y}}$ & $\mathrm{t}_{\mathrm{x}} \mathrm{t}_{\mathrm{x}}$ & Fully faithful for all inputs (with respect to voicing). \\
& $\mathrm{t}_{\mathrm{x}} \mathrm{s}_{\mathrm{x}}$ & $\mathrm{t}_{\mathrm{x}} \mathrm{z}_{\mathrm{y}}$ & $\mathrm{t}_{\mathrm{x}} \mathrm{s}_{\mathrm{x}}$ & [-voice] consonants are in correspondence with each \\
& $\mathrm{s}_{\mathrm{x}} \mathrm{t}_{\mathrm{x}}$ & $\mathrm{s}_{\mathrm{x}} \mathrm{d}_{\mathrm{y}}$ & $\mathrm{s}_{\mathrm{x}} \mathrm{t}_{\mathrm{x}}$ & other; otherwise, non-correspondence. \\
& $\mathrm{s}_{\mathrm{x}} \mathrm{s}_{\mathrm{x}}$ & $\mathrm{s}_{\mathrm{x}} \mathrm{z}_{\mathrm{y}}$ & $\mathrm{s}_{\mathrm{x}} \mathrm{s}_{\mathrm{x}}$ & \\
\hline \multirow{4}{*}{ F.noc } & $\mathrm{t}_{\mathrm{x}} \mathrm{t}_{\mathrm{y}}$ & $\mathrm{t}_{\mathrm{x}} \mathrm{d}_{\mathrm{y}}$ & $\mathrm{t}_{\mathrm{x}} \mathrm{t}_{\mathrm{y}}$ & Fully faithful for all inputs (with respect to voicing). \\
& $\mathrm{t}_{\mathrm{x}} \mathrm{s}_{\mathrm{y}}$ & $\mathrm{t}_{\mathrm{x}} \mathrm{z}_{\mathrm{y}}$ & $\mathrm{t}_{\mathrm{x}} \mathrm{s}_{\mathrm{y}}$ & No correspondence between any consonants in any \\
& $\mathrm{s}_{\mathrm{x}} \mathrm{t}_{\mathrm{y}}$ & $\mathrm{s}_{\mathrm{x}} \mathrm{d}_{\mathrm{y}}$ & $\mathrm{s}_{\mathrm{x}} \mathrm{t}_{\mathrm{y}}$ & outputs. \\
& $\mathrm{s}_{\mathrm{x}} \mathrm{s}_{\mathrm{y}}$ & $\mathrm{s}_{\mathrm{x}} \mathrm{z}_{\mathrm{y}}$ & $\mathrm{s}_{\mathrm{x}} \mathrm{s}_{\mathrm{y}}$ & \\
\hline \multirow{5}{*}{ Dis.noc } & $\mathrm{t}_{\mathrm{x}} \mathrm{d}_{\mathrm{y}}$ & $\mathrm{t}_{\mathrm{x}} \mathrm{d}_{\mathrm{y}}$ & $\mathrm{t}_{\mathrm{x}} \mathrm{d}_{\mathrm{y}}$ & All inputs with two [-voice] consonants undergo \\
& $\mathrm{t}_{\mathrm{x}} \mathrm{z}_{\mathrm{y}}$ & $\mathrm{t}_{\mathrm{x}} \mathrm{z}_{\mathrm{y}}$ & $\mathrm{t}_{\mathrm{x}} \mathrm{z}_{\mathrm{y}}$ & dissimilation: one consonant surfaces as [+voice], \\
& $\mathrm{s}_{\mathrm{x}} \mathrm{d}_{\mathrm{y}}$ & $\mathrm{s}_{\mathrm{x}} \mathrm{d}_{\mathrm{y}}$ & $\mathrm{s}_{\mathrm{x}} \mathrm{d}_{\mathrm{y}}$ & averting the need for surface correspondence. \\
& $\mathrm{s}_{\mathrm{x}} \mathrm{z}_{\mathrm{y}}$ & $\mathrm{s}_{\mathrm{x}} \mathrm{z}_{\mathrm{y}}$ & $\mathrm{s}_{\mathrm{x}} \mathrm{z}_{\mathrm{y}}$ & No distinctions made among forms with voicing \\
& $\mathrm{d}_{\mathrm{x}} \mathrm{t}_{\mathrm{y}}$ & & $\mathrm{d}_{\mathrm{x}} \mathrm{t}_{\mathrm{y}}$ & disagreement: all forms with one [+voice] C and one \\
& $\mathrm{d}_{\mathrm{x}} \mathrm{s}_{\mathrm{y}}$ & & $\mathrm{d}_{\mathrm{x}} \mathrm{s}_{\mathrm{y}}$ & [-voice] C are equally good outputs of dissimilation. \\
& $\mathrm{z}_{\mathrm{x}} \mathrm{t}_{\mathrm{y}}$ & & $\mathrm{z}_{\mathrm{x}} \mathrm{t}_{\mathrm{y}}$ & Inputs with voiced consonants are fully faithful. \\
& $\mathrm{z}_{\mathrm{x}} \mathrm{s}_{\mathrm{y}}$ & & $\mathrm{z}_{\mathrm{x}} \mathrm{s}_{\mathrm{y}}$ &
\end{tabular}

The three different languages in the typology are the only possible patterns generated by this system of $\mathrm{ABCD}$ constraints. Two of these languages, 'F.cor' and 'F.noc', are fully faithful languages: they have no input-output disparities (modulo the co-optima that differ only in [ \pm sibilant] - a feature that none of these three constraints are sensitive to). The difference between the two faithful languages lies only in the surface 
correspondence structure of certain types of inputs. In the F.cor language, pairs of voiceless consonants have surface correspondence with one another - hence the name 'F.cor', abbreviating the pattern of faithful and "stable" correspondence in these inputs. This is not to say that all outputs have surface correspondence, though: the input $/ \mathrm{t} \mathrm{z} /$ illustrates this. The only CORR constraint in this subsystem refers to [-voice]; candidates with correspondence between voiced and voiceless segments are included in GEN, but they are harmonically bounded because no constraint favors this kind of superfluous correspondence. As such, /t $\mathrm{z} /$ surfaces with faithful non-correspondence, as $\left[\begin{array}{ll}t_{x} & z_{y}\end{array}\right]$ (or its equivalent) irrespective of constraint ranking. The language F.noc differs from F.cor in that it treats voiceless-voiceless pairs the same way: they surface faithfully, with two [-voice] consonants, and no correspondence between them.

The language of more direct interest is Dis.noc. The grammar of this language produces dissimilation: inputs with two voiceless consonants surface unfaithfully, with one of them becoming voiced, such that inputs like /t $t /$ (can) map to $\left[\begin{array}{ll}t_{x} & d_{y}\end{array}\right]$. The extremely simple nature of the constraint set results in a large bundle of co-optima: none of the three constraints in this simple sub-system care which consonant dissimilates (nor do they care whether segments are faithful for [ \pm sibilant]). This language exhibits dissimilation of voicelessness - schematically on par with what we find in Kinyarwanda. ${ }^{2}$

The ranking conditions of each language are given in (4) below. Worth noting here is that these are the only possibilities that emerge from this subsystem: it is simple enough to understand in its entirety in a few short pages. We turn now to the second ABCD subsystem under consideration.

Rankings for each language in the typology of 2rt VlessDiss

\begin{tabular}{|c|c|c|}
\hline Dis.noc & F.cor & F.noc \\
\hline CC.EdGE $(\sigma)$ CoRR $[-$-voice $]$ & IDENT(voice) CC.EdGE( $(\sigma)$ & IDENT(voice) CORR $\cdot[$-voice $]$ \\
\hline IDENT(voice) & $\searrow_{\text {Corr } \cdot[- \text {-voice }]}$ & CC.EdGe $(\sigma)$ \\
\hline
\end{tabular}

\section{2rt_SibHarm: typology of a 4-constraint ABCD subsystem}

The second simple ABCD subsystem we consider here is one designed to produce sibilant harmony. This adds a dimension of complexity above the 2rt_VlessDiss system: it's crucial to refer to two features, not just one. This is in keeping with the Agreement By Correspondence interpretation of harmony: CORR $\cdot[+$ sibilant] points to sibilants as the class of segments that participate in similarity-based agreement, and CC.IDENT(voice) requires them to agree for a feature that they don't necessarily share already.

The full typology of the 2rt_SibHarm system is given in (5). As with the 2rt_VlessDiss subsystem, the range of possibilities is small - as is the number of inputs needed to demonstrate them.

Four languages constitute the typology of the 2rt_SibHarm system. Two are entirely familiar from the 2rt_VlessDiss system, labeled again with the familiar names F.cor and F.noc. These are fully faithful, and differ only in the distribution of surface correspondence in optima. Also as before, the schematic characterization as having correspondence or not is only manifested for certain inputs. For $/ \mathrm{s} \mathrm{s} /$, for instance, there is no reason not to have faithful correspondence: this input is perfect, in that it has two sibilants that agree in voicing already, so having correspondence cannot incur any penalty. Similarly, no constraint favors correspondence in inputs with only one sibilant, like $/ z$ t/, so these invariably surface as faithful and non-correspondent.

The interesting meat of the typology comes from inputs with disagreeing sibilants: $/ s \quad z /$ and $/ z \quad s /$. Two grammars (F.cor and F.noc) map such inputs faithfully. The other two, Har.cor and Dis.noc, show us harmony and dissimilation, shaded in pink and yellow, respectively. In the harmony language, sibilants correspond, and are adjusted to agree for voicing (in one way or the other - none of the constraints of this system have a preference for one direction or one feature value over the other). In the dissimilation language, we find the same situation of 'unstable' correspondence repaired in the opposite way: by

\footnotetext{
${ }^{2}$ A difference from Kinyarwanda is that this dissimilation obtains between [-voice] Cs that straddle a syllable boundary, rather than those that straddle the stem boundary. This might put it more on par with a case like that found in Bakairi (Wetzels \& Mascaró 2001:235ff).
} 
changing one sibilant into a non-sibilant, such that no correspondence is needed - and, accordingly, no agreement is necessary.

(5) Typology of 2rt_SibHarm

\begin{tabular}{|c|c|c|c|c|}
\hline$\downarrow$ Lgs $\backslash$ Inputs $\rightarrow$ & $\mathbf{s} \mathbf{s}$ & $\mathbf{s} \quad \mathbf{z}$ & $z \quad t$ & Remarks \\
\hline Har.cor & $\mathrm{S}_{\mathrm{x}} \mathrm{S}_{\mathrm{x}}$ & $\begin{array}{l}s_{x} s_{x} \\
z_{x} z_{x}\end{array}$ & $z_{x} t_{y}$ & $\begin{array}{l}\text { Harmony: sibilants must correspond, and must agree } \\
\text { for }[ \pm \text { voice }]\end{array}$ \\
\hline Dis.noc & $\mathrm{S}_{\mathrm{x}} \mathrm{S}_{\mathrm{x}}$ & $\begin{array}{l}t_{x} z_{y} \\
s_{x} d_{y}\end{array}$ & $z_{x} t_{y}$ & $\begin{array}{l}\text { Dissimilation: sibilants that disagree in [ } \pm \text { voice }] \\
\text { dissimilate (satisfying CORR } \cdot[+ \text { sibilant }] \text { 'vacuously') }\end{array}$ \\
\hline F.cor & $\mathrm{s}_{\mathrm{x}} \mathrm{s}_{\mathrm{x}}$ & $\mathrm{s}_{\mathrm{x}} \mathrm{z}_{\mathrm{x}}$ & $z_{x} t_{y}$ & $\begin{array}{l}\text { Fully faithful for all inputs. Sibilants always } \\
\text { correspond with each other. }\end{array}$ \\
\hline F.noc & $s_{x} s_{x}$ & $\mathrm{~s}_{\mathrm{x}} \mathrm{z}_{\mathrm{y}}$ & $z_{x} t_{y}$ & $\begin{array}{l}\text { Fully faithful for all inputs. Sibilants correspond if } \\
\text { and only if they agree on }[ \pm \text { voice }] \text {. }\end{array}$ \\
\hline
\end{tabular}

The typology of this system, though still quite simple, already gives an impression of structure and parallelism. The languages Har.cor and F.cor share the trait of having correspondence between all pairs of sibilants. Similarly, in the languages Dis.noc and F.noc, consonants correspond only if they agree in voicing in the input (i.e. if CC.IDENT(voice) is satisfied by a fully faithful mapping). Unintuitively, though, these extensional traits do not come with any formal similarity in the grammar; we can see this from the ranking structures in (6). The behavior of each language is determined solely by which of the four constraints is ranked on the bottom; ranking among other three constraints is irrelevant. Consequently, there are no elementary rankings conditions shared between the grammars of these classes. ${ }^{3}$

(6) Rankings for each language in the typology of 2rt VlessDiss

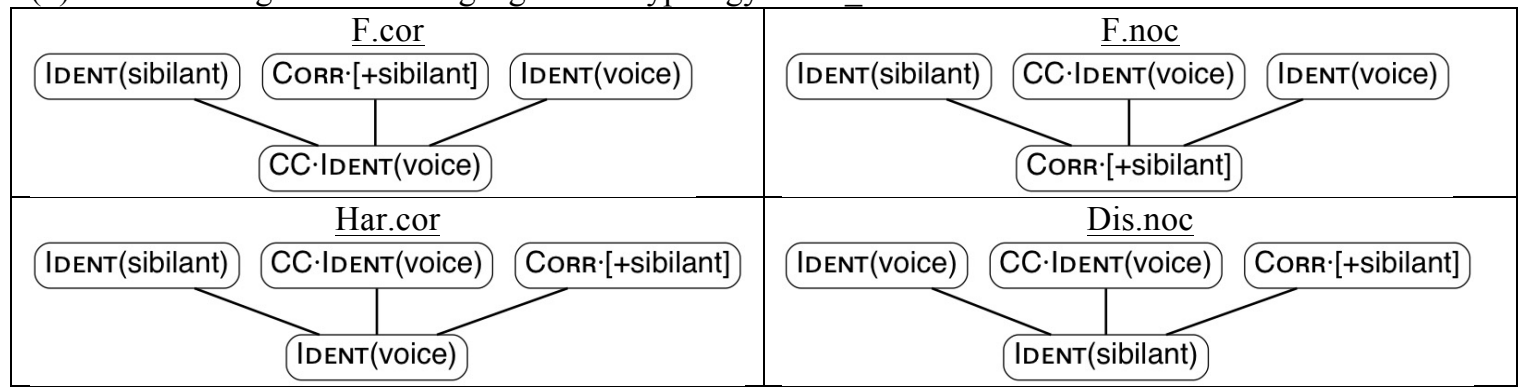

The impression of structure in the typology of 2rt_SibHarm sets up an expectation that free interaction of the two ABCD subsystems should have an internal structure like its components, which we turn to now.

\section{The combined 2 rt2f typology, with interacting subsystems}

The system $2 \mathrm{rt} 2 \mathrm{f}$ consists of all the constraints of both the 2rt_SibHarm and 2rt_VlessDiss subsystems, for a total of 6 constraints, assessing all of the inputs and candidates defined in section 2 above. The typology consists of 16 languages, listed in (7) below. The five inputs shown here illustrate all the distinctions evident in the whole typology; all other possible inputs were considered, none show patterns of cross-linguistic difference different than the ones shown here. The rows show all 16 languages, with their mappings for each of the five inputs in the colored cells. The two rightmost rows indicate the disposition of each half of the system.

Echoes of the simpler systems leap out immediately. Out of the 16 languages, the first 12 are

\footnotetext{
${ }^{3}$ Merchant \& Prince (2015: $\left.\$ 4.2 .1\right)$ discuss this kind of formal relationship among 'bots' - ranking structures in which the behavior of the system depends solely on the bottom constraint, with no shared ERCs.
} 
straightforwardly the product of the two simpler systems. Thus, each possible disposition of the VlessDiss side of the system is further refined into four distinct languages, reflecting the four possible configurations of the SibHarm side of the system.

(7) Typology of combined 2rt 2f system

\begin{tabular}{|c|c|c|c|c|c|c|c|}
\hline$\downarrow$ Lgs $\backslash$ Inputs $\rightarrow$ & t t & S $\mathbf{S}$ & $\begin{array}{ll}\mathbf{S} \quad \mathbf{Z}\end{array}$ & $\begin{array}{ll}\mathbf{Z} \quad \mathbf{Z}\end{array}$ & $z \quad t$ & VlessDiss & SibHarm \\
\hline $\operatorname{Lg} \# 1$ & $t_{x} t_{x}$ & $\mathrm{~S}_{\mathrm{x}} \mathrm{S}_{\mathrm{x}}$ & $\begin{array}{l}S_{x} s_{x} \\
z_{x} z_{x}\end{array}$ & $\mathrm{z}_{\mathrm{x}} \mathrm{z}_{\mathrm{x}}$ & $z_{x} t_{y}$ & f.cor & har.cor \\
\hline Lg \#2 & $t_{x} t_{x}$ & $\mathrm{~S}_{\mathrm{x}} \mathrm{S}_{\mathrm{x}}$ & $\begin{array}{l}t_{x} z_{y} \\
s_{x} d_{y}\end{array}$ & $\mathrm{z}_{\mathrm{x}} \mathrm{z}_{\mathrm{x}}$ & $\mathrm{z}_{\mathrm{x}} \mathrm{t}_{\mathrm{y}}$ & f.cor & dis.noc \\
\hline $\operatorname{Lg~\# 3}$ & $t_{x} t_{x}$ & $\mathrm{~s}_{\mathrm{X}} \mathrm{s}_{\mathrm{X}}$ & $\mathrm{s}_{\mathrm{x}} \mathrm{z}_{\mathrm{x}}$ & $\mathrm{z}_{\mathrm{x}} \mathrm{z}_{\mathrm{x}}$ & $z_{x} t_{y}$ & f.cor & f.cor \\
\hline $\operatorname{Lg} \# 4$ & $t_{x} t_{x}$ & $\mathrm{~S}_{\mathrm{X}} \mathrm{S}_{\mathrm{X}}$ & $\mathrm{s}_{\mathrm{x}} \mathrm{z}_{\mathrm{y}}$ & $z_{x} z_{x}$ & $z_{x} t_{y}$ & f.cor & f.noc \\
\hline $\mathrm{Lg} \# 5$ & $t_{x} t_{y}$ & $\mathrm{~S}_{\mathrm{x}} \mathrm{S}_{\mathrm{x}}$ & $\begin{array}{l}S_{x} S_{x} \\
z_{x} z_{x}\end{array}$ & $\mathrm{z}_{\mathrm{x}} \mathrm{z}_{\mathrm{x}}$ & $\mathrm{z}_{\mathrm{x}} \mathrm{t}_{\mathrm{y}}$ & f.noc & har.cor \\
\hline $\operatorname{Lg~\# 6}$ & $\mathrm{t}_{\mathrm{x}} \mathrm{t}_{\mathrm{y}}$ & $\mathrm{S}_{\mathrm{x}} \mathrm{S}_{\mathrm{X}}$ & $\begin{array}{l}t_{x} z_{y} \\
s_{x} d_{y}\end{array}$ & $\mathrm{z}_{\mathrm{x}} \mathrm{Z}_{\mathrm{x}}$ & $\mathrm{z}_{\mathrm{x}} \mathrm{t}_{\mathrm{y}}$ & f.noc & dis.noc \\
\hline Lg \#7 & $t_{x} t_{y}$ & $\mathrm{~s}_{\mathrm{X}} \mathrm{s}_{\mathrm{X}}$ & $\mathrm{s}_{\mathrm{X}} \mathrm{z}_{\mathrm{X}}$ & $\mathrm{z}_{\mathrm{X}} \mathrm{z}_{\mathrm{X}}$ & $\mathrm{z}_{\mathrm{x}} \mathrm{t}_{\mathrm{y}}$ & f.noc & f.cor \\
\hline $\operatorname{Lg} \# 8$ & $t_{x} t_{y}$ & $\mathrm{~S}_{\mathrm{x}} \mathrm{S}_{\mathrm{x}}$ & $\mathrm{S}_{\mathrm{x}} \mathrm{z}_{\mathrm{y}}$ & $z_{x} z_{x}$ & $z_{x} t_{y}$ & f.noc & f.noc \\
\hline $\operatorname{Lg~\# 9}$ & $\begin{array}{l}t_{x} d_{y} \\
d_{x} t_{y} \\
\end{array}$ & $\mathrm{~S}_{\mathrm{x}} \mathrm{S}_{\mathrm{x}}$ & $\begin{array}{l}s_{x} s_{x} \\
z_{x} z_{x} \\
\end{array}$ & $\mathrm{z}_{\mathrm{x}} \mathrm{z}_{\mathrm{x}}$ & $\mathrm{z}_{\mathrm{x}} \mathrm{t}_{\mathrm{y}}$ & dis.noc & har.cor \\
\hline $\operatorname{Lg} \# 10$ & $\begin{array}{l}t_{x} d_{y} \\
d_{x} t_{y} \\
\end{array}$ & $\begin{array}{l}t_{x} z_{y}, s_{x} d_{y} \\
d_{x} s_{y}, z_{x} t_{y}\end{array}$ & $\begin{array}{l}t_{x} z_{y} \\
s_{x} d_{y} \\
\end{array}$ & $\begin{array}{l}d_{x} z_{y} \\
z_{x} d_{y}\end{array}$ & $\mathrm{z}_{\mathrm{x}} \mathrm{t}_{\mathrm{y}}$ & dis.noc & dis.noc \\
\hline Lg \#11 & $\begin{array}{l}t_{x} d_{y} \\
d_{x} t_{y}\end{array}$ & $\mathrm{~S}_{\mathrm{x}} \mathrm{S}_{\mathrm{x}}$ & $\mathrm{s}_{\mathrm{x}} \mathrm{z}_{\mathrm{x}}$ & $\mathrm{z}_{\mathrm{x}} \mathrm{z}_{\mathrm{x}}$ & $\mathrm{z}_{\mathrm{x}} \mathrm{t}_{\mathrm{y}}$ & dis.noc & f.cor \\
\hline $\operatorname{Lg} \# 12$ & $\begin{array}{l}t_{x} d_{y} \\
d_{x} t_{y} \\
\end{array}$ & $\begin{array}{l}S_{x} z_{y} \\
z_{x} S_{y} \\
\end{array}$ & $\mathrm{~s}_{\mathrm{x}} \mathrm{z}_{\mathrm{y}}$ & $z_{x} z_{y}$ & $z_{x} t_{y}$ & dis.noc & f.noc \\
\hline $\operatorname{Lg} \# 13$ & $t_{x} t_{x}$ & $\mathrm{~S}_{\mathrm{x}} \mathrm{S}_{\mathrm{x}}$ & $\begin{array}{l}t_{x} z_{y} \\
s_{x} d_{y} \\
\end{array}$ & $\begin{array}{l}d_{x} z_{y} \\
z_{x} d_{y}\end{array}$ & $z_{x} t_{y}$ & f.cor & dis.noc* \\
\hline $\operatorname{Lg~\# 14}$ & $t_{x} t_{x}$ & $\mathrm{~S}_{\mathrm{X}} \mathrm{S}_{\mathrm{x}}$ & $\mathrm{S}_{\mathrm{x}} \mathrm{Z}_{\mathrm{y}}$ & $\mathrm{z}_{\mathrm{x}} \mathrm{z}_{\mathrm{y}}$ & $z_{x} t_{y}$ & f.cor & f.noc* \\
\hline $\operatorname{Lg} \# 15$ & $t_{x} t_{y}$ & $\begin{array}{l}t_{x} s_{y} \\
s_{x} t_{y}\end{array}$ & $\begin{array}{l}t_{x} z_{y} \\
s_{x} d_{y} \\
\end{array}$ & $\begin{array}{l}d_{x} z_{y} \\
z_{x} d_{y}\end{array}$ & $\mathrm{z}_{\mathrm{x}} \mathrm{t}_{\mathrm{y}}$ & f.noc & dis.noc* \\
\hline Lg \#16 & $t_{x} t_{y}$ & $S_{x} S_{y}$ & $S_{x} z_{y}$ & $z_{x} z_{y}$ & $z_{x} t_{y}$ & f.noc & f.noc* \\
\hline
\end{tabular}

Somewhat less obvious in character are the last four languages. These show a subset of the same fundamental types of effects as the first 12: all inputs show faithful correspondence, faithful noncorrespondence, or dissimilation of [+sibilant]. What sets them apart is the relative distribution of these types of mappings. For example, compare languages \#2 and \#13. Both involve the same kinds of mappings: pairs of voiceless consonants are mapped faithfully, with correspondence, and sibilants undergo dissimilation. The difference is that $1 \mathrm{~g}$. \#2 applies dissimilation only to inputs with disagreeing sibilants; lg. \#13 also shows dissimilation for two [+voice] sibilants: $/ \mathrm{z} \quad \mathrm{z} / \rightarrow\left[\begin{array}{ll}\mathrm{d}_{\mathrm{x}} & \mathrm{z}_{\mathrm{y}}\end{array}\right]\left(=\left[\begin{array}{ll}\mathrm{z}_{\mathrm{x}} & \mathrm{d}_{\mathrm{y}}\end{array}\right]\right)$.

(8) Two ways to get sibilant dissimilation a. Lg. \#2

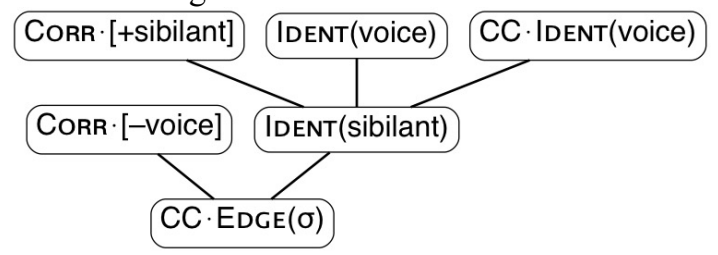

b. Lg. \#13

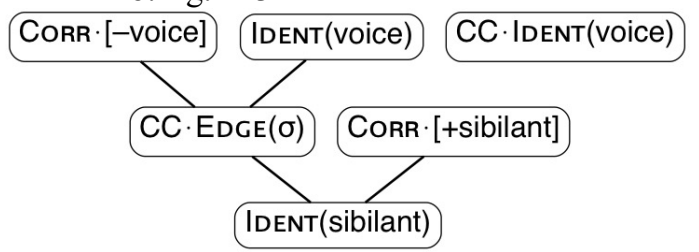

Scrutiny of the ranking structures reveals a key insight about these two grammars: dissimilation happens in both, but arises in different ways. Consider the first two strata of the ranking for lg. \#2, on the left in (8): we see the ranking CORR $\cdot[+$ sibilant], CC·IDENT(voice), IDENT(voice) $\gg$ IDENT(sibilant). This is exactly the 
same ranking condition responsible for the sibilant dissimilation pattern in the 2rt_SibHarm subsystem (6d). The resultant dissimilation also has exactly the same distribution: sibilants dissimilate (one becoming a stop) if and only if they disagree in voicing. Focusing on the constraints of the 2rt_VlessDiss system, we again see a very familiar structure, this time from the 2rt_VlessDiss subsystem: CORR $[$-voice], $\operatorname{IDENT}($ voice) $\gg \mathrm{CC} \cdot \operatorname{EDGE}(\sigma)$. In the simpler subsystem, this ranking produced faithful correspondence between [-voice] Cs, as it does here. The result: lg. \#2 properly contains one of the grammars from each of the simpler subsystems; it is straightforwardly their product.

Turning to the ranking for $1 \mathrm{~g}$. \#13 on the right in (8), we see the same configuration of the 2rt_VlessDiss constraints: CORR $\cdot[-$ voice], IDENT(voice) $\gg \mathrm{CC} \cdot \operatorname{EDGE}(\sigma)$. Faithful correspondence among voiceless Cs arises in precisely the same way here as in lg. \#2, and in the F.cor language in the VlessDiss subsystem. The constraints of the SibHarm subsystem, on the other hand, are in a novel arrangement. The bottom of this ranking structure is CORR $[+$ sibilant], CC.EDGE $(\sigma) \gg$ IDENT(sibilant). This exact ranking condition is not familiar from either of the simpler cases considered above, because it involves constraints drawn from both of the simpler CON systems. However, the structural arrangement of these constraints is transparently similar to the ranking that produces voiceless dissimilation in the VlessDiss subsystem: CORR $\cdot[\mathrm{F}], \mathrm{CC} \cdot$ EDGE $\gg$ IDENT(F). This ranking favors dissimilation between any sibilants that straddle a syllable edge - irrespective of their voicing. This is why $/ z \quad z /$ undergoes dissimilation here, instead of emerging with faithful correspondence as in lg. \#2. (The reason that $/ \mathrm{s} \mathrm{s} /$ does not dissimilate is because the other constraints that refer to [-voice] are ranked so as to produce faithful correspondence: CORR $\cdot[$-voice], IDENT(voice) $\gg \mathrm{CC} \cdot \operatorname{EDGE}(\sigma)$.)

The result illustrated here is that combining the two simple sub-systems yields a CON that includes a third subsystem, consisting of $\{$ CORR $\cdot[+$ sibilant], CC·EDGE $(\sigma)$, IDENT(sibilant)\}. The interactions between these three constraints are immediately obvious: by themselves, they exhibit exactly three possibilities, completely homologous with the $2 \mathrm{rt}$ _VlessDiss system. As such, the combined $2 \mathrm{rt} 2 \mathrm{f}$ typology offers two ways to moderate the interactions between sibilants. Their disposition of correspondence can be handled by the constraints of the 2rt_SibHarm system, in which case they interact on the basis of voicing, and we find a split between inputs that have disagreeing sibilants vs. inputs with agreeing ones. Alternatively, the behavior of sibilants can be moderated by the constraints of this "emergent" subsystem, in which case they interact on the basis of straddling a syllable boundary.

The typology of the combined $2 \mathrm{rt} 2 \mathrm{f}$ system doesn't have the structure of a simple $3 \mathrm{x} 4$ product of the two simple subsystems. Rather, it is the product of three such subsystems, listed in (9). Each of the component subsystems sits at the lower limit for internal complexity: each consists of exactly one CORR constraint, one CC constraint that operates on correspondence, and faithfulness constraints for the feature(s) picked out by those constraints.

Constraint subsystems contained in CON_2rt2f

\begin{tabular}{|l|l|l|l|}
\cline { 2 - 4 } \multicolumn{1}{c|}{} & 2rt_VlessDiss & 2rt_SibHarm & 2rt_SibDiss (emergent) \\
\hline CORR constraint & CORR $\cdot[-$ voice $]$ & CORR $\cdot[+$ sibilant $]$ & CORR $\cdot[+$ sibilant $]$ \\
\hline CC $\cdot$ constraint & CC $\cdot$ EDGE $(\sigma)$ & CC $\cdot$ IDENT $($ voice $)$ & CC $\cdot$ EDGE $(\sigma)$ \\
\hline IO faithfulness & IDENT(voice $)$ & IDENT(voice), IDENT(sibilant) & IDENT(sibilant) \\
\hline Typology size & 3 permutations & 4 permutations & 3 permutations \\
\hline
\end{tabular}

The intuited size of the resulting typology $(3 \times 4 \times 3=36)$ is restricted because the constraint sets comprising each subsystem overlap: the subsystems cannot be permuted freely because some permutations involve conflicting ranking conditions. For example, the emergent subsystem, '2rt_SibDiss', has exactly three possible configurations, parallel to the 2rt_VlessDiss subsystem. One of these leads to faithful correspondence: CORR $\cdot[+$ sibilant], IDENT(sibilant) $\gg \operatorname{CC} \cdot \operatorname{EDGE}(\sigma)$; the other two have the consequence that sibilants in different syllables never correspond. If sibilants in different syllables are prohibited from corresponding, it follows that the constraints of the 2rt_SibHarm system can make no further distinctions. The distinction between F.noc and F.cor emerges only through the different treatments of sibilants that agree in voicing vs. those that don't. But if all sibilants are prohibited from corresponding, independent of voicing, this difference cannot emerge. The result: rankings among the SibHarm constraints matter only if the SibDiss subsystem is configured in one specific way. To arrive at a deeper understanding of these kinds 
of interactions in the ranking conditions, we turn now to a more formal analysis of the structures of the properties and their genesis in ranking structure.

\section{Formal properties of the typologies}

In analyzing the deeper structure of the $2 \mathrm{rt} 2 \mathrm{f}$ typology, and its sub-components, we draw on the concepts and notation of Alber, DelBusso \& Prince (2015), and Merchant \& Prince (2015). Chief among these is the notion of typological properties, defined as sets of mutually incompatible ranking conditions. These are notated here as ' $\mathrm{C} 1<>\mathrm{C} 2$ ', where $\mathrm{C} 1$ and $\mathrm{C} 2$ are constraints, and the operator ' $<>$ ' means 'either (a) $\mathrm{C} 1 \gg$ $\mathrm{C} 2$, or (b) $\mathrm{C} 2 \gg \mathrm{C} 1$ '. These two contradictory ranking relations are termed the two values of the property. The idea derives a parameter-esque choice between two grammars, or sets of grammars. One value is the ranking condition $\mathrm{C} 1 \gg \mathrm{C} 2$; this defines a class of languages in the typology, all of which share the extensional consequences that follow from this ranking. The other value of the property, $\mathrm{C} 2 \gg \mathrm{C} 1$, is a contradictory ranking that defines a distinct and non-overlapping set of grammars, which have in common a different set of extensional consequences that follow from this different ranking condition.

6.1 2rt_VlessDiss The three languages of the 2rt_VlessDiss typology can be impressionistically characterized in terms of faithfulness and correspondence. ${ }^{4}$ Exactly one language is unfaithful, the one named 'Dis.noc' in (3) above; the other two, 'F.cor' and 'F.noc' are fully faithful. The only distinction between these is the surface correspondence structure of (certain) faithful outputs: whether voiceless Cs that fail to dissimilate do so by tolerating non-correspondence, or by tolerating correspondence that violates $\mathrm{CC} \cdot \operatorname{EDGE}(\sigma)$.

These two extensional traits of the resulting languages come hand-in-hand with particular configurations of CON. If the surface correspondence constraints both dominate IDENT, the result is dissimilation (the grammar of Dis.noc). If IDENT dominates one of the SCorr constraints, the result is one of the two fully faithful languages. The choice between them depends on which of the SCorr constraints is the lower one. To refer to structural positions in this disjunctive kind of situation, we employ the notation 'sub', as a function that picks out the subordinate (=lowest-ranked) of a subset of constraints: " $\left\{\right.$ CORR $\cdot[- \text { voice], CC·EdGE }(\sigma)\}_{-}$sub" refers to the lower of these two constraints (whichever it may be).

The typological choice between dissimilation and faithfulness is a direct consequence of the relative ranking of IDENT and the subordinate of the two surface correspondence constraints (10a). ${ }^{5}$ Within the class of faithful languages defined by (10a), a further division exists, between faithful correspondence and faithful non-correspondence. This choice comes down to the relative ranking between the two SCorr constraints (10b). Only the lower of the two correspondence constraints needs to be violated to avoid dissimilation; as such, the ranking between these constraints dictates which of the two species of faithful candidates is optimal. The resulting typological structure is illustrated in (11).

Typological properties of 2rt_VlessDiss

a. Dissimilating / Faithfulness: $\{\mathrm{CC} \cdot \operatorname{EDGE}(\sigma), \operatorname{CORR} \cdot[-\mathrm{voice}]\} \_$sub $<>\operatorname{IDENT}($ voice $)$

b. Correspondent / Non-correspondent (among Faithful lgs): CORR $\cdot[-$ voice $]<>C C \cdot \operatorname{EDGE}(\sigma)$

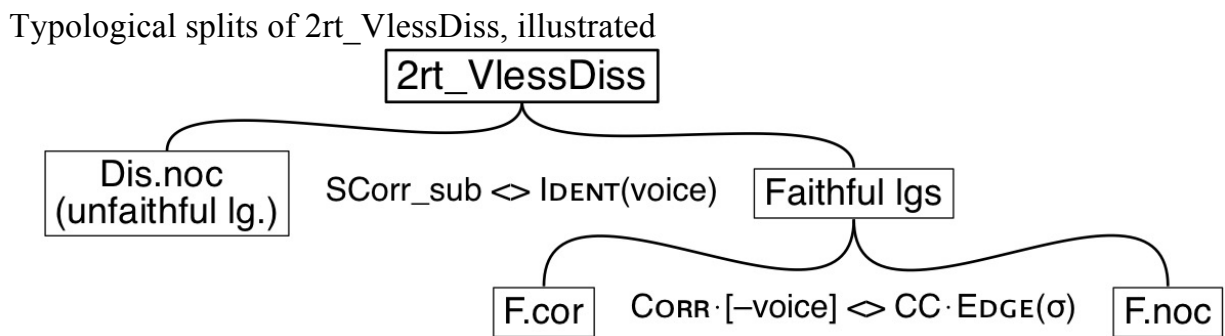

\footnotetext{
${ }^{4}$ Note that there is no a priori basis to order these splits: each separates one language in the VlessDiss typology from the other two.

${ }^{5}$ Characterizing this in terms of the lower of the two SCorr constraints here entails that both dominate faithfulness.
} 
6.2 2rt_SibHarm In the 2rt_SibHarm subsystem, the typology is only slightly more complex. Here, the extensional traits cross-classify. F.cor and Har.cor are both languages where sibilants always correspond; the distinction is whether there is harmony among correspondents. Likewise, Har.cor and Dis.noc both share the trait of mapping some inputs unfaithfully: they are both grammars that prohibit disagreement between sibilants, and differ only in how that prohibition is enforced (assimilation vs. dissimilation). The result is that the typology has a symmetrical structure. The first cut, between the fully faithful languages and the unfaithful ones, is a consequence of whether the bottom constraint is one of the two I-O faithfulness constraints, or one of the surface correspondence constraints. That is, the choice between these sets of languages is dictated by the relative ranking of the lower constraint of each type IDENT_sub and SCorr_sub (12a). If the constraint on the bottom is an IO faithfulness constraint, the result is either harmony or dissimilation (depending on which of the IO IDENT constraints it is). If the bottom constraint is one of the SCorr constraints, then the result is one of the fully faithful languages.

The remaining two properties in (12) make the finer distinctions within each class of languages which follow from relative rankings among each class of constraint. Among the unfaithful languages, the ranking of the two IO IDENT constraints determines whether agreement is enforced by harmony, or by dissimilation (12b): the type of unfaithful mapping chosen will be whichever violates the lower-ranked faithfulness constraints. Thus, among grammars that involve unfaithfulness, IDENT(voice) 》 IDENT(sibilant) characterizes the dissimilating language, while IDENT(sibilant) 》 IDENT(voice) characterizes harmony.

Among the two fully faithful languages, the distinguishing trait is how agreement fails - whether it's a lack of correspondence, or whether disagreement between correspondents is tolerated. This choice comes down to the ranking of the two SCorr constraints (12c). If CORR $[+$ sibilant $] \gg C C \cdot I D E N T($ voice), then disagreeing sibilants will correspond, but not harmonize. Alternatively, if CC.IDENT(voice) 》 CORR $\cdot[+$ sibilant], then agreement will be vacuously enforced, by simply not having correspondence between disagreeing sibilants. The treeoid diagram in (13) illustrates the structure of this typology, and how these choices between mutually-exclusive ranking conditions fit together.

Typological properties of 2rt_SibHarm

a. Unfaithful / Faithful:

$\{\text { CC·IDENT(voice), CORR } \cdot[+ \text { sibilant }]\}_{-}$sub $<>\{\text {IDENT(voice), IDENT(sibilant) }\}_{-}$sub

b. Harmony / Dissimilation: (among Unfaithful languages defined by (a)) IDENT(voice) $<>$ IDENT(sibilant)

c. Correspondent / Non-correspondent: (among Faithful languages defined by (a)) CORR $\cdot[+$ sibilant $]<>$ CC $\cdot \operatorname{IDENT}($ voice)

Typological splits of 2rt_SibHarm, illustrated

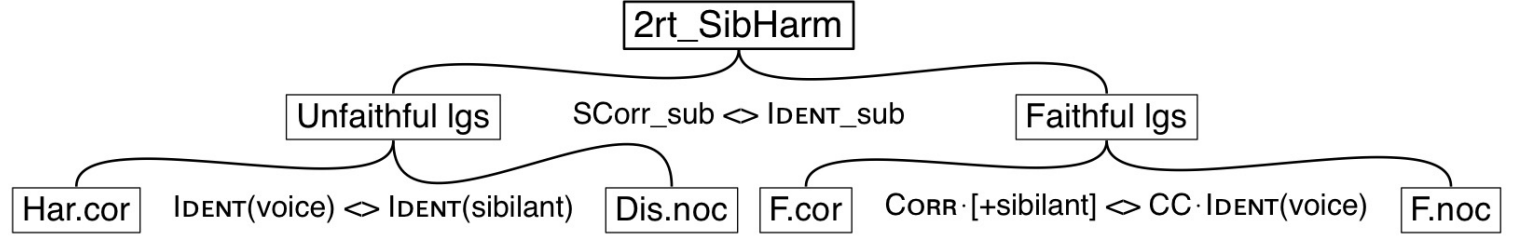

6.3 2rt2f: the combined system The typology of the combined system contains, properly, the typologies of both of its component subsystem. This is laid bare by analyzing the combined typology into properties in the same way demonstrated above with its simpler components. The typological properties of the combined $2 \mathrm{rt} 2 \mathrm{f}$ system are listed in (14). Each language in the typology is characterized by a distinct combination of values on these properties, in the same way as in the simpler typologies considered above. Moreover, the specific properties of the two simpler typologies can be observed, transparently, in the $2 \mathrm{rt} 2 \mathrm{f}$ typology. The behavior of voiceless consonants is subject to exactly the same two nested choices as in the 2rt_VlessDiss component system: (14a-b) are exactly the same choices between ranking conditions as seen in (10) above. The properties of the 2rt_SibHarm system likewise emerge in exactly the same way: the 
same properties from (12) above recur here (14e-g), with the same scope relations among them.

What makes the combined system distinct from the straightforward product of its components are the two properties in (14c) and (14d). These are structurally homologous with the 2rt_VlessDiss subsystem: they involve the interaction of $\mathrm{CC} \cdot \operatorname{EDGE}(\sigma)$ with one CORR constraint and one (IO) IDENT constraint, which both pick out the same feature (albeit [+sibilant] rather than [-voice]). These permit the same three types of patterns seen in the voiceless dissimilation side of the system: they produce dissimilation, or faithfulness with correspondence tolerated, or faithfulness with non-correspondence. These three configurations govern the behavior of sibilants that straddle the edge of a syllable - again, in the same fashion as the interaction of $\mathrm{CC} \cdot \operatorname{EdgE}(\sigma)$ with $\operatorname{CORR} \cdot[-\mathrm{voice}]$ and IDENT(voice) govern the behavior of voiceless consonants that straddle a syllable edge.

The two properties in (14c-d) take scope over the properties from the 2rt_SibHarm subsystem. The sibilant harmony subsystem controls the role of voicing agreement between sibilants. However, if sibilants are forced to dissimilate for reasons independent of CC IDENT, then there is no finer distinction to be made on the basis of voicing. By the same token, if the correspondence structure of faithful sibilants is dictated independent of the constraints responsible for the sibilant harmony subsystem, then there is no possibility for voicing agreement to make distinctions in terms of correspondence structure. So, if the subsystem of $\mathrm{CC} \cdot \operatorname{EdgE}(\sigma), \operatorname{CORR} \cdot[+$ sibilant$]$, and IDENT(sibilant), is configured to prohibit any correspondence between sibilants in different syllables, then the two dimensions of the 2rt_SibHarm typology collapse. The treeoid diagram in (15) illustrates these scope relations.

(14) Typological properties of $2 \mathrm{rt} 2 \mathrm{f}$

i. $\quad$ Properties governing voiceless $C s$

a. Voicelessness: Dissimilating / Faithful

$\{\mathrm{CC} \cdot \operatorname{EDGE}(\sigma), \operatorname{CORR} \cdot[-$ voice $]\} \_$sub $<>\operatorname{IDENT}($ voice $)$

b. Faithful voiceless Cs: Correspondent / Non-correspondent (only for Faithful value of (a)) CORR $\cdot[+$ sibilant $]<>\mathrm{CC} \cdot \operatorname{EDGE}(\sigma)$

ii. $\quad$ Properties governing sibilant co-occurrence

c. Correspondence between sibilants in different syllables: Impossible / Allowed

$\mathrm{CC} \cdot \operatorname{EdGE}(\sigma)<>\{\operatorname{IDENT}($ sibilant $), \operatorname{CORR} \cdot[+$ sibilant $]\} \_$sub

d. Mapping of sibilants: Faithful / Dissimilation (only if correspondence is Impossible on (c)) IDENT(sibilant) $<>$ CORR $\cdot[+$ sibilant $]$

iii. Properties governing sibilant agreement

e. Disagreeing sibilants: Unfaithful / Faithful (only if correspondence is Allowed by (c))

$\{$ CC $\cdot$ IDENT(voice), CORR $\cdot[+$ sibilant $]\} \_$sub $<>\{\text {IDENT(voice), IDENT(sibilant) }\}_{-}$sub

f. Enforcement of sibilant agreement: Harmony / Dissimilation (only if Unfaithful on (e)) IDENT(voice) $<>$ IDENT(sibilant)

g. Correspondent / Non-correspondent: (only if Faithful on (e))

CORR $\cdot[+$ sibilant $]<>$ CC $\cdot$ IDENT(voice) 
Treeoid of sibilant-related properties

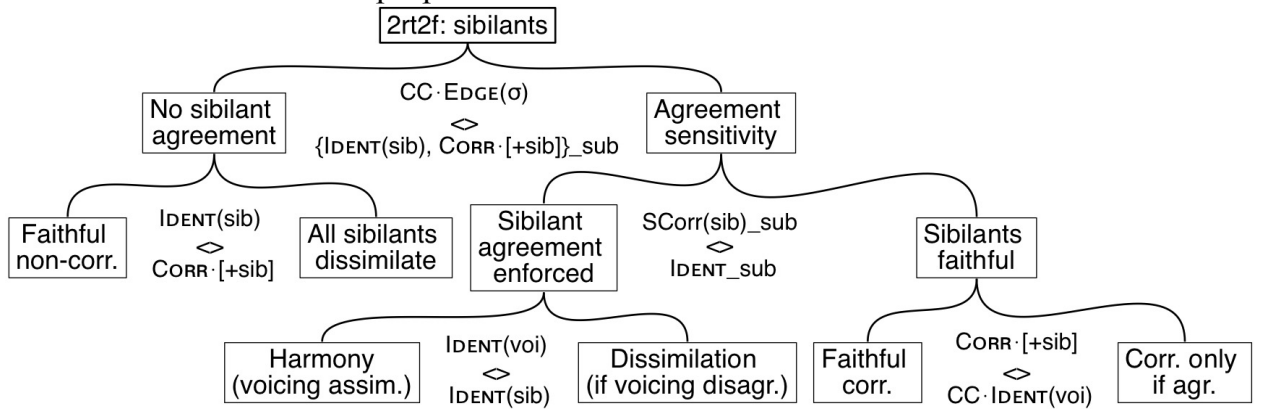

The relative ranking among the constraints that comprise the 2rt_VlessDiss side of the typology is not in a dependency relation with the constraints that can affect the behavior of sibilants. There is no dependency between how a language handles voiceless consonants generally, and how it handles sibilants: in addition to making the property-based choices illustrated in (15), each language also has values on the two properties in (14a-b), and these choices are fully distinct.

The table in (16) shows the property values for each language in the $2 \mathrm{rt} 2 \mathrm{f}$ typology.

(16) Property values of the 16 languages in the 2rt2f typology

\begin{tabular}{|c|c|c|c|c|c|c|c|}
\hline \multirow[t]{2}{*}{ Lg. } & \multicolumn{2}{|c|}{ Voiceless Cs (2rt_VlessDiss) } & \multicolumn{2}{|c|}{ Sibilants (2rt_SibDiss) } & \multicolumn{3}{|c|}{ Harmony (2rt_SibHarm) } \\
\hline & Faith & $\begin{array}{l}\text { Voiceless corr. } \\
\text { across } \sigma \mathrm{s}\end{array}$ & $\begin{array}{l}\text { Sibilant corr. } \\
\text { across } \sigma \mathrm{s}\end{array}$ & $\begin{array}{l}\text { Sibilant } \\
\text { mapping }\end{array}$ & Faith & $\begin{array}{l}\text { Enforcement } \\
\text { of agreement }\end{array}$ & $\begin{array}{l}\text { Disharmonic } \\
\text { sibilant corr. }\end{array}$ \\
\hline 1 & Faithful & Corresp. & Allowed & & Unfaithful & Harmony & \\
\hline 2 & Faithful & Corresp. & Allowed & & Unfaithful & Dissim. & \\
\hline 3 & Faithful & Corresp. & Allowed & & Faithful & & Corresp. \\
\hline 4 & Faithful & Corresp. & Allowed & & Faithful & & Non-corresp. \\
\hline 13 & Faithful & Corresp. & Impossible & Dissim. & & & \\
\hline 14 & Faithful & Corresp. & Impossible & Faithful & & & \\
\hline 5 & Faithful & Non-corresp. & Allowed & & Unfaithful & Harmony & \\
\hline 6 & Faithful & Non-corresp. & Allowed & & Unfaithful & Dissim. & \\
\hline 7 & Faithful & Non-corresp. & Allowed & & Faithful & & Corresp. \\
\hline 8 & Faithful & Non-corresp. & Allowed & & Faithful & & Non-corresp. \\
\hline 15 & Faithful & Non-corresp. & Impossible & Dissim. & & & \\
\hline 16 & Faithful & Non-corresp. & Impossible & Faithful & & & \\
\hline 9 & Dissim. & & Allowed & & Unfaithful & Harmony & \\
\hline 11 & Dissim. & & Allowed & & Faithful & & Corresp. \\
\hline 10 & Dissim. & & Impossible & Dissim. & & & \\
\hline 12 & Dissim. & & Impossible & Faithful & & & \\
\hline
\end{tabular}

\section{Concluding remarks}

Our aim in this paper has been to solve the typological ramifications of interactions between different subsets of $\mathrm{ABCD}$ constraints. We have undertaken the first step of this problem by reducing it to one of its simplest types of cases: the interaction of two subsystems, each consisting of a small number of constraints, operating on a limited (but exhaustively considered) space of possible inputs and candidates. This structure mirrors a real practical concern for an analyst: the possibility that correspondence-based analyses of two patterns in the same language might clash with one another. The finer details of the models chosen here are also firmly grounded in the reality of Kinyarwanda - a language that exhibits both sibilant harmony and voiceless dissimilation (Bennett 2015, and sources therein). From understanding this simple case, 
preliminary understandings of generalities of such interactions start to emerge.

The major results are summarized as follows. We observe that the whole of a typology is bigger than the sum of its parts: free interaction of two constraint subsystems can give rise to new effects beyond the product of the components that comprise it. We also note that these emergent effects are quite limited in nature. In the case considered here, the result is limited to exactly one new type of dissimilation system: a language that has dissimilation across syllable edges, rather than just to enforce voicing agreement. Moreover, this result is intuitively predictable: it is the product of correspondence from one of the component systems, together with a restriction on correspondence taken from the other. The interaction can also be understood as an emergent subsystem of exactly the same irreducibly simple character as the 2rt_VlessDiss and 2rt_SibHarm components. This points to a bigger generality: that when multiple tuples of CORR, CC.Limiter and faithfulness constraints can freely interact, the range of new effects they produce is of the simplest sort (equivalent in structure to one such tuple) - and that further and less intuitive asymmetries in the typology emerge from distinctions made by one tuple being dependent on specific configurations of others.

\section{References}

Alber, Birgit and Alan Prince. Forthcoming. Typologies. Ms., University of Verona and Rutgers University. Alber, Birgit, Natalie DelBusso and Alan Prince. 2015. From intensional properties to universal support. ROA-1235.

Bennett, Wm. G. 2015. The Phonology of Consonants: Harmony, Dissimilation and Correspondence. Cambridge: CUP.

Hansson, Gunnar Ó. 2007. Blocking effects in agreement by correspondence. Linguistic Inquiry 38.2:395-409.

Hansson, Gunnar Ó. 2010. Consonant Harmony: Long-Distance Interaction in Phonology. UC Press, Berkeley, CA.

Hansson, Gunnar Ó. 2014. (Dis)Agreement by (Non)Correspondence: Inspecting the foundations. In UC Berkeley Phonology Lab annual report, 2014, 3-62.

Iacoponi, Luca. 2016. The typology of Headed Agreement By Correspondence. In Proceedings of Phonology 2015, UBC/Simon Fraser University, Vancouver.

Inkelas, Sharon and Stephanie S. Shih. 2014. Unstable surface correspondence as the source of local conspiracies. NELS proceedings 44:191-204.

Merchant, Nazarré and Alan Prince. 2015. The Mother of All Tableaux. Ms, Eckerd College and Rutgers University.

Mester, R. Armin. 1986. Studies in Tier Structure. Ph.D. thesis, University of Massachusetts, Amherst.

Prince, Alan, Bruce Tesar and Nazarré Merchant. 2015. OTWorkplace. Software version X_88. Available from https://sites.google.com/site/otworkplace/.

Rose, Sharon and Rachel Walker. 2004. A typology of consonant agreement as correspondence. Language, 80:475531.

Shih, Stephanie S. and Sharon Inkelas. 2014. A subsegmental correspondence approach to contour tone (dis)harmony patterns. In John Kingston et al. (eds.). Proceedings of Phonology 2013. University of Massachusetts, Amherst.

Walker, Rachel. 2000a. Yaka nasal harmony: Spreading or segmental correspondence? BLS proceedings 26:321-332.

Walker, Rachel. 2000b. Long-distance consonantal identity effects. WCCFL proceedings 19:532-545.

Walker, Rachel. 2015. Feature-Restricted Evaluation of Surface Identity. Paper presented at the 2015 LSA annual meeting, Portland, January 2015.

Wetzels, W. Leo and Mascaró, Joan. 2001. The Typology of Voicing and Devoicing. Language 77.2:207-244.

Yip, Moira. 1988. The Obligatory Contour Principle and Phonological Rules: A Loss of Identity. Linguistic Inquiry 19:65-100. 